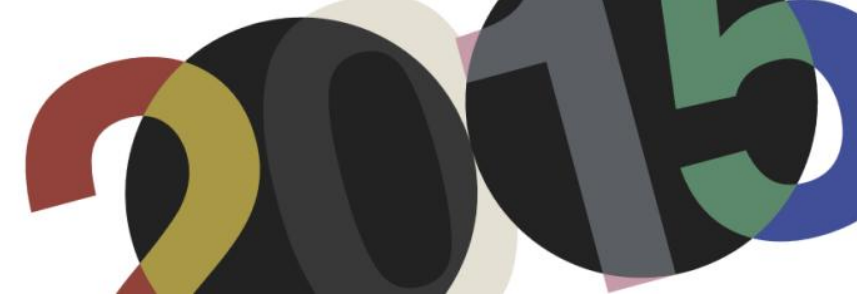

DOI: http://dx.doi.org/10.4995/LC2015.2015.845

\title{
Autour du pyjama de Le Corbusier Le vêtement comme modèle de pensée fondateur
}

\author{
C. Félix-Fromentin \\ Lacth, Ecole Nationale Supérieure d'Architecture et de Paysage de Lille
}

\begin{abstract}
Résumé: Je m'intéresse, depuis le territoire du design, à l'intérêt que Le Corbusier porta pour le vêtement moderne, à partir d'une conférence de 1929 où la réforme du vêtement l'emporte à fonder son concept d' "équipement domestique » pour la «machine à habiter». La richesse du modèle renvoie vers des écrits préalables, notamment sa théorie des "objets-membres artificiels" qui s'est trouvée soutenir une nouvelle définition "prothétique » du design, et par ailleurs des témoignages vécus plus intimes qui amènent un point de vue alternatif et dynamique sur la relation corporelle aux artefacts modernes. Au cœur des débats sur la place et le rôle de la machine industrielle, la position de Le Corbusier révèle sa complexité et sa singularité, qui s'éclaire grâce à des travaux et des concepts propres à l'époque, telle que la Rythmique et la phénoménologie du mouvement, les notions d'euvre vivante, de sensation et de mesure. Cela emporte à esquisser une conception prothétique corbuséenne, paradoxale, offrant en retour d'enrichir la compréhension de cette idéologie encore très active, et aussi de contribuer à une théorie du vêtement en design, si frêle sur le sujet.
\end{abstract}

Resumen: Me interesa, desde el territorio del diseño, el interés que Le Corbusier trajo a la prenda moderna, desde una conferencia de 1929 en que la reforma de la prenda le lleva a instaurar su concepto de "equipo del hogar" por la "máquina para vivir". La riqueza del modelo se refiere a los escritos anteriores, especialmente su teoría de la "extremidades objetos artificiales", que se encontró sostener una nueva definición "prótesis" del diseño , y por otro lado, testimonios vividos más íntimos que conducen a un punto de vista alternativo y dinámico sobre relación cuerpo a los artefactos modernos. En el centro del debate sobre el lugar y el papel de la máquina industrial, la posición de Le Corbusier revela su complejidad y singularidad, que se ilumina gracias a las obras y los conceptos específicos de la época, tal como la Rítmica y la fenomenología de movimiento, las nociones de obras vivas, de sensación y de medida. Esto lleva a esbozar un diseño protésico corbuseano, paradójico, permitiendo a cambio enriquecer la comprensión de esta ideología que aun sigue muy activa, y también para contribuir a una teoría de la vestimenta en diseño, tan frágil sobre este tema.

Abstract: I focus, from the territory of design, on the interest Le Corbusier brought to the modern garment, from a 1929 conference where the dressreform leads him to develop his concept of "équipement domestique" for the "machine à habiter". Richness of the model refers to prior writings, especially his theory of "objets-membres artificiels", which occured supporting a new "prosthetic" definition of design, and besides that, intimate testimonies that develop an alternative point of view, more dynamic, about body relationship to modern artefacts. At the heart of debates of the time about the place and role of industrial machinery, the position Le Corbusier holds reveals its complexity and uniqueness, which can be clarify through works and concepts specific of that time such as Eurythmics and the phenomenology of movement, notions of living work of art, sensation and measure. This encourages suggest a Corbusean, paradoxical, prosthetic design, allowing in return enrich the understanding of this ideology still very active, and also to contribute to a theory of dress in design, so slight on this subject.

Mots-clé: Le Corbusier, design, vêtement, prothétique, Rythmique.

Palabras clave: Le Corbusier, diseño, prenda, prótesis, Rítmica.

Keywords: Le Corbusier, design, clothing, prosthetic, Eurythmics. 


\section{Introduction}

«Avec Le Corbusier chez un chemisier où il désire acheter un pyjama.

-Je voudrais un pyjama sombre, uni, de forte couleur, sans revers, sans bordure à filet blanc.

-Nous n'avons pas cela Monsieur. Nous n'avons qu'avec filet blanc.

Le Corbusier proteste :

-Vous, les chemisiers, vous n'avez pas encore inventé un pyjama seyant, pour qu'on n'ait pas toujours l'air d'être dans une chambre à coucher de théâtre!

-Monsieur, on les fait toujours comme cela.

Même réponse avenue de l'Opéra, boulevard Saint Michel, etc... ${ }^{1}$

Ce souvenir de Jean Petit, relaté dans Le Corbusier parle _non daté, quoique le sujet traité le situe au-delà des années 1940_ semble d'abord une parabole comme celle du cordonnier qui sert à Adolf Loos dans Ornement et Crime $^{2}$ à appeler à un dépassement de l'ornement issu de l'artisanat traditionnel. Si la tonalité s'y apparente en effet, la scène est toutefois possiblement plus authentique, et ceci pour deux raisons. Elle évoque la réalité d'une mutation profonde au début du XXème siècle dans le secteur de l'habillement visant à se débarrasser des codes culturels et sociaux qui imposent aux corps leur tenue ; le pyjama fut un article vestimentaire représentatif, porté en extérieur comme en vêtement d'intérieur informel ${ }^{3}$, par les femmes comme par les hommes, qui suscita beaucoup d'engouement et de créativité.

Par ailleurs, nous reconnaissons à Le Corbusier la qualité et pertinence de ses confessions, témoins de l'intrication de la pensée et de la vie capables de contrebalancer positivement ses textes plus rhétoriques. Comme Paul Valery y invita à l'occasion de son premier cours de Poétique au Collège de France en 1937, sur les questions les plus vastes et complexes, afin de demeurer dans des «proportions humaines », mieux vaut s'en tenir à sa propre expérience vécue et observée scrupuleusement. "Je m'efforce de n'oublier jamais que chacun est la mesure des choses ${ }^{4}$.

Ainsi, ce qui apparaît comme un souvenir trivial n'en est sans doute pas un, et justifie sa citation dans le livre de Petit, d'autant plus que Le Corbusier précise in fine à son sujet : "J'ai écrit quelque part, vers 1929 : tant que le costume n'aura pas été transformé, la révolution ne sera jamais faite. » La transformation du vêtement est annoncée de la sorte comme préalable à la révolution de l'architecture qui allait l'agiter toute sa vie.

Cette assertion suscite la curiosité et invite à fouiller ses textes en quête d'explications. Dans son ouvrage White walls : designer dresses, the fashioning of modern architecture, Mark Wigley s'est en quelque sorte attelé à la tâche quand il s'est interrogé sur le silence historique et théorique qui entourait les murs blancs caractéristiques de l'architecture moderne, dont l'architecte suisse fut le promoteur principal. Son étude, exhaustive et précieuse,

\footnotetext{
${ }^{1}$ Petit, Jean : Le Corbusier parle. Paris : Editions Forces Vives, 1967. p. 53

${ }^{2}$ LOOS Adolf, « Ornement et crime ». In Paroles dans le vide - Malgré tout. Paris : Editions Ivrea, 1994. p.206. Cet essai fut publié par la revue L'Esprit Nouveau.

${ }^{3}$ Le pyjama, en hindi «vêtement de jambe », sorte de large pantalon souvent accompagné d'une kurta (tunique fendue), arrive en France au début du siècle. Réinterprété (notamment par Gabrielle Chanel, Paul Poiret, Sonia Delaunay ou Elsa Schiaparelli) et assorti à une veste-chemise, il est porté dans les années 1920 comme costume idoine aux nouvelles pratiques sportives ou balnéaires. Il ne fut employé comme costume de nuit dans le vestiaire masculin qu'à partir des années 1940, en remplacement de la chemise de nuit ou liquette.

${ }^{4}$ Valéry, Paul : Première Leçon du cours de poétique. In Oeuvres I, Paris : La Pléiade, 1957. p. 1358.
} 
est parvenue à mettre à jour derrière eux un indéniable «principe (moderne) du vêtement " $^{5}$, inhérent à la modernité, quoique effacé voire défendu par l'histoire canonique.

Je m'intéresse pour ma part à Le Corbusier depuis le territoire de ce que l'on appelle aujourd'hui en français très généralement le design, et dans le cadre d'un questionnement sur cet artefact particulier qu'est le vêtement. Car s'il tourmenta le mouvement moderne, l'historiographie du design l'a étonnamment délaissé.

Connexe à l'architecture notamment via le problème des arts décoratifs et la question de l'habitation, l'architecte a côtoyé le milieu du design en émergence, et participé aux actions et débats qui l'agitèrent. Toutefois, sa position est qualifiée d'ambiguë, d'une manière générale par Alexandra Midal dans Design, L'anthologie, plus particulièrement par Reyner Banham dans Théorie et Design à l'ère industrielle (discutant la pertinence de ses « machines » vis-à-vis de l'outil industriel), ou encore par Adrian Forty dans Industrial Design and Prosthesis (où il apparaît comme père malgré lui d'une théorie «prothétique » du design). La visée de cette réflexion peut dès lors s'énoncer ainsi : l'intérêt singulier de Le Corbusier pour le vêtement pourrait-il apporter quelques clartés sur son legs au design?

Dans un premier temps, nous analyserons un texte de 1929 intitulé «L'aventure du mobilier », où le vêtement féminin moderne fonde son concept d' « équipement domestique » pour la « machine à habiter ». Dans un second temps, des écrits préalables sur le sujet, dans L'art décoratif d'aujourd'hui d'une part, dans des écrits plus intimes d'autre part, amèneront à approfondir le modèle lui-même, et à y déceler une problématique constitutive, dont nous explorerons dans un troisième temps les conséquences sur sa conception du design, en nous appuyant sur des témoignages plus tardifs parus dans Quand les cathédrales étaient blanches.

\section{De la réforme du vêtement à la réforme du mobilier - Le vêtement moderne féminin comme machine à s'habiller}

L'appel à la révolution vestimentaire, dont Le Corbusier se remémore avec Petit, se rapporte en réalité à une conférence qu'il a tenue à Buenos Aires le 19 octobre 1929, dont le texte fut publié en 1930 dans Précisions sur un état présent de l'architecture et de l'urbanisme. Il y prédit «L'aventure du mobilier » à partir de celle qu'à vécue le vêtement moderne féminin et ses effets constatés sur la vie quotidienne.

«La femme nous a précédés. Elle a réalisé la réforme de son costume. Elle s'était trouvée dans cette impasse : suivre la mode et renoncer alors à l'apport des techniques modernes, à la vie moderne. (...)

Suivre la mode : elle ne pouvait se mêler d'auto ; elle ne pouvait ni prendre son métro ou son autobus, ni agir avec célérité au bureau ou au magasin. Pour réaliser la construction quotidienne de sa toilette : coiffure, bottes, boutonnage de la robe, elle n'aurait plus eu le temps de dormir.

Alors la femme a coupé ses cheveux, et ses jupes, et ses manches. Elle s'en va tête nue, bras nus, jambes libres. Et en cinq minutes, elle s'habille. Et elle est belle : elle nous séduit du charme de ses grâces dont les couturiers ont admis de tirer parti.

Le courage, l'entrain, l'esprit d'invention avec lesquels la femme a opéré sa révolution vestimentaire sont un miracle des temps modernes. Merci! »

\footnotetext{
${ }^{5}$ Référence au «principe du revêtement » de Gottfried Semper, réactualisé par Adolf Loos.

${ }^{6}$ Le Corbusier, «L'aventure du mobilier». In Précisions sur un état présent de l'architecture et de l'urbanisme. Paris : Edition Altamira, 1994. p. 106-107
} 
Ce constat enthousiaste est opposé sans attendre, dans le registre masculin, aux habits d'apparat encore empesés, empanachés, non adaptés au travail, ne permettant pas de concilier élégance et caractère pratique. Seul le «mécano» se démarque selon l'auteur, qui appelle dès lors à ce que «l'esprit de réforme » ici constaté se répande à tous les actes de la vie. Selon Banham, le jeune architecte est pourtant réputé pour sa panoplie de l'homme moderne, vêtements sombres, chapeau melon, noeud papillon. Le Corbusier est lui-même plus retenu quand il justifie le choix de son costume anglais comme une étape de transition, qui, à défaut d'être moderne, a au moins cet effet important de le «neutraliser », car : «Il est utile d'arborer un aspect neutre en ville ${ }^{7}$.

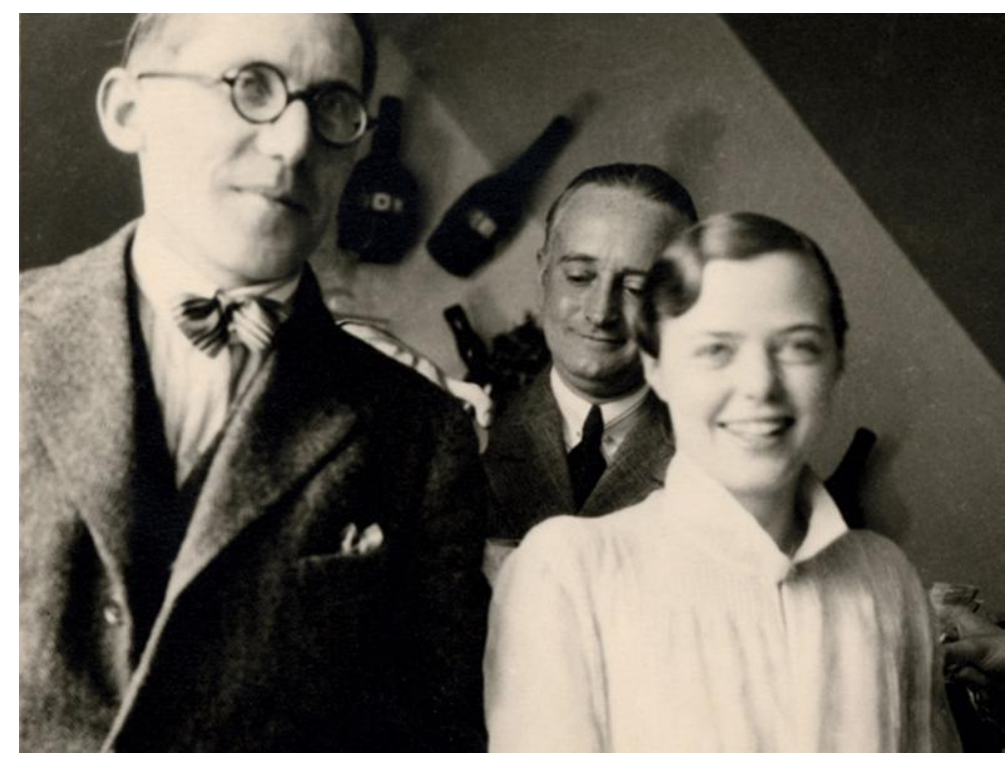

1. Le Corbusier, Pierre Jeanneret, Charlotte Perriand en 1927 CFLC/ADAGP

Pour bien saisir de quoi il est question, il est utile de poser le contexte historique de cette révolution.

Comme cela fut abondamment étudié ${ }^{8}$, les positions corbuséeens prendraient source au cœur de la Lebensreform, vaste contexte de remise en cause esthétique, sociale, politique au tournant du XIXème au XXème siècle. Dans une inspiration nietzschéenne et une méfiance vis à vis du progrès, elle est animée par une quête d'harmonie avec le milieu naturel, intéressée par la compréhension des phénomènes de perception et de cognition, et nourrie diversement des théories psychophysiques, de psychologie expérimentale, ou d'esthétique scientifique en plein développement.

Cette rénovation de la vie fait écho à une réforme du vêtement engagée dans la seconde moitié du siècle précédent visant à affranchir le vêtement de l'arbitraire de la mode, et selon les cas, à lui appliquer les préceptes de l'hygiénisme, à le penser selon des critères de rationalité, honnêteté ou moralité, à servir l'aspiration au Gesammtkunstwerk et l'introduction de l'art dans tous les domaines de la vie. Wigley en relate finement les origines et développements dans les milieux des arts décoratifs et de l'architecture, notons simplement que cette mobilisation fut soutenue activement par Henry van de Velde que l'on sait avoir été lu et apprécié par Le Corbusier. La dynamique qu'il initia fut très suivie, Frank Lloyd Wright ou Peter Behrens par exemple y ont

\footnotetext{
${ }^{7}$ Ibid. p. 107

${ }^{8}$ L'exposition au Centre Pompidou de Paris du 29 avril au 3 août 2015 et son catalogue Le Corbusier - Mesures de l'homme commentent abondamment cet ancrage. On peut y lire par exemple sur le sujet Migayrou, Fréderic : «Les yeux dans les yeux. Architecture \& Mathesis ». In Cinqualbre, Olivier; Migayrou, Fréderic (Dir.) : Le Corbusier - Mesures de l'homme, Paris : Editions du Centre Pompidou, 2015. p. 14.
} 
participé en concevant des vêtements dans le cadre de leurs projets. Avant la première guerre mondiale, les avant-gardes artistiques, tant cubistes que futuristes et suprématistes, furent concernés activement à leur tour par cette œuvre portée ou «peinture vivante ${ }^{9}$ comme nouveau champ d'investigation poétique. Paris, en tant que laboratoire de l'avant-garde, par ailleurs capitale de la mode, centralisait les énergies sur le sujet. Alors qu'il y séjournait, celui qui était encore Charles-Edouard Jeanneret fréquenta Paul Poiret et fut introduit dans son cercle. Amédée Ozenfant était pour sa part en affaire dans la mode avec la sœur du couturier. Au sortir de la guerre, en accord avec l'assagissement général et la volonté désormais concrète de construire un monde nouveau à l'ère de la machine devenue omniprésente, les préoccupations autour du vêtement concourent au développement de typologies vestimentaires nouvelles adaptées aux conditions de la vie moderne, dont Le Corbusier se fait le porte-parole dans sa conférence.

Le «mécano» dont il vante l'esprit de réforme fait sans doute référence à la Tuta, conçue par le futuriste Thayaht en 1919. «Tuta d'un pezzo » ${ }^{10}$ (tout d'une seule pièce), il s'agit d'une combinaison intégrale aux contours géométriques en forme de T comme le T-Type Shirt, vêtement universel destiné aux hommes, femmes et enfants pour être porté en toutes occasions. Il serait inspiré du overall américain, à qui Loos prédit la même année une destinée formidable ${ }^{11}$. Equipé de nombreuses poches, agréments pratiques nouvellement attenants au vêtement qui débarrassent des sacoches, il illustre bien cette remarque de l'architecte : «La poche, les poches devraient être la clé de voûte du vêtement moderne $»^{12}$.

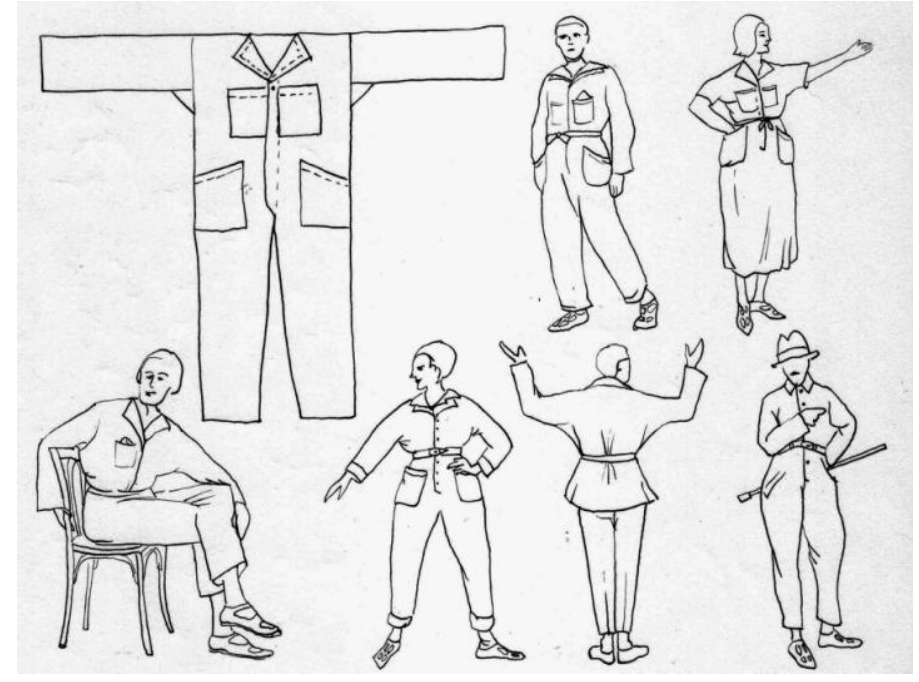

2. La Tuta et ses déclinaisons, 1919, d'après les dessins de Thayaht

Le vestiaire féminin évoqué semble être celui élaboré par Poiret, voire Chanel pour qui « le vêtement doit bouger sur le corps $»^{13}$. Pour celle qui s'opposait au luxe des grands couturiers qui confondent costume de théâtre et vêtement pour le quotidien, seul un travail studieux de simplification des formes et de dépouillement, accompagné d'une connaissance pratique du corps vivant, ("...je m'attaque au mannequin vivant, alors que les

\footnotetext{
${ }^{9}$ La formule est de Robert Delaunay au sujet des vêtements conçus par Sonia. Delaunay, Robert : Du cubisme à l'art abstrait, Paris : Edition SEVPEN, 1958.

${ }^{10}$ Benhamou, Viviana : «Ernesto Thayaht (1893 - 1953), nouvelles perspectives ». In Guillaume, Valérie (Dir) : Europe 1910-1933, Quand l'art habillait le vêtement. Paris : Edition Paris Musées, 1997. p. 44.

${ }^{11}$ Loos, Adolf : «Réponses à des questions du public ». in Paroles dans le vide - Malgré Tout. Op.cit. p.267.

12 «L'aventure du mobilier». Op.cit. p. 107.

${ }^{13}$ Morand, Paul : L'allure de Chanel, Paris : Herman, 1976. p. 52.
} 
autres dessinent, font des poupées ou des maquettes... ${ }^{14}$ ) parvint à atteindre des lignes contemporaines, sans taille, défiant les genres, et agrémentées de poches. Il en résulte une épure de vêtement, qui supporte bien ce commentaire extrait du manifeste du Purisme : «statique, générale, expressive de l'invariant, mais ni accidentelle, ni exceptionnelle, ni impressionniste, ni inorganique, ni protestataire, ni pittoresque ${ }^{15}$. Un objet puriste donc, qu'il s'agit dès lors de transposer au problème du mobilier puis à l'habitation.

Tout d'abord, comme les anciens habits ont opéré leur renouvellement en adoptant une certaine universalité en terme de besoins, fonctions et dimensions, il appelle le mobilier à gagner les qualités du standard. Synonyme de quotidien, régulier, constant, similaire ou de côte moyenne, il peut combler nos «besoins d'hommes » ${ }^{16}$, quand par ailleurs il satisfait à l'esprit de l'époque «ingénié » par la fabrication industrielle vertueuse en terme de «précision, efficacité, pureté des formes et des lignes». Comme Banham le rapporte, on sait que le jeune architecte, alors en mission d'étudier l'avancée des allemands en matière de design, fut initié par Hermann Muthesius notamment à la théorie des types et à la défense du standard ; notions très proches, le type évoque un universel qui s'actualise en le standard dans le contexte technique de la production de masse.

\section{«Fonctions standard,}

besoins standard,

objets standard,

dimensions standard. ${ }^{17}$

De là, le vêtement standard, par sa construction générique et géométrale qui peut être rapportée au type poche, va inspirer l'idée de casier. "Je vais affirmer qu'en dehors des sièges et des tables, les meubles ne sont à vrai dire que des casiers. »

«Dispositif moderne » qui permet, à l'instar des poches, de disposer toutes nos choses, elles-mêmes de tailles proportionnées à nos membres et adaptés à nos gestes, observe-t-il, le casier standard satisfait à la situation industrielle. Il autorise l'architecte ou le particulier à élaborer un ensemble cohérent et architectonique en les disposant soit contre les murs, soit indépendamment, faisant office de cloison pleine ou partielle. L'organisation optimisée libère de la place, et fait ainsi gagner de l'espace et du temps.

Dès lors, «il reste à équiper l'intérieur des casiers ${ }^{18}$. Or, si les caissons sont des parallélépipèdes semblables, les solutions pour les aménager au mieux en regard de leur destination sont multiples et variées, qui peuvent aller «du simple apparat» au «raffinement le plus complet» (portes, tiroirs, étagères, tablettes, niches, ...). Le générique accueille d'autant mieux la diversification et la personnalisation.

L'analogie au vêtement moderne est ici encore possible, et riche de problématiques parce que non tranchée. Individualité versus standard : la dualité fait en effet écho à une dispute célèbre opposant en 1914 van de Velde à Muthesius au sein du Deutscher Werkbund. Le Corbusier n'y fait pas explicitement allusion dans sa conférence, mais sa solution pour les casiers indique sa position dans le débat. Alors que le premier soutient la cause d'une certaine expression artistique dans la conception vestimentaire, le second prône une standardisation complète

\footnotetext{
${ }^{14}$ Ibid.

${ }^{15}$ Jeanneret, Charles-Edouard; OZENFANT, Amédée : Après le cubisme. Paris : Éditions des Commentaires, 1918. Fin d'ouvrage non paginé.

${ }^{16}$ «L'aventure du mobilier ». Op.cit. p. 109.

17 « L'aventure du mobilier». Op.cit. p. 110.

${ }^{18}$ Ibid. p. 116.
} 
immédiate $^{19}$. Cette controverse va rapidement concerner toute production industrielle, en reléguant comme mineur le problème du vêtement. Au Bauhaus, Walter Gropius suivit d'abord le point de vue de van de Velde, puis se rallia à celui de son protagoniste. Au sein de l'établissement, à la «Bauhaustracht» (tenue du bauhaus), de coupe simplifiée pouvant être fabriquée dans des textiles variés, succéda la combinaison constructiviste aux nombreuses poches fonctionnelles, portée par Moholy-Nagy notamment, avant que Gropius n'impose le costume-type, le concepteur nouveau devant porter (corporellement) sa nouvelle orientation industrielle.

Le sujet fut toutefois ravivé par Lilly Reich en 1922 en quête d'une voie intermédiaire. Consciente de la nécessité de sortir cet objet utile des domaines de l'art et de l'artisanat, élitistes, et de le relocaliser sur le terrain de l'industrie où le secteur économique de la mode a de toutes façons la mainmise et n'hésite pas à contrefaire la facture manuelle, elle soutient toutefois la particularité de cet artefact qui appelle une « organic unity» ${ }^{20}$ avec la personne qui le porte, pour ce que cela participe à élever «the expression of the soul and his feeling of being alive ». Pour cela, elle propose que soient définis les types, ou patrons génériques, du vestiaire moderne, formes simples que des broderies par exemple viendraient personnaliser. Les artistes ou proto-designers réagirent nombreux à la polémique. La Tuta citée plus haut, modèle d'invariance vestimentaire, est ouverte en réalité à plusieurs variantes, tel que les illustrations diffusées à l'occasion de sa publication y invitent : en plus de décliner l'étoffe, on peut choisir entre manche longue ou courte, version robe, et on est invité à la porter avec une ceinture ou un cordon noué, sur une chemise ou à même la peau. Elle sera imaginée en Bi-tuta (costume en deux pièces) et Tuta termica (version pour l'hiver), révélant la richesse de la typisation initiale.

Une dernière allusion aux possibilités offertes par le vêtement moderne au profit du mobilier, hormis les casiers, concerne les sièges, pour l'attitude ou la «tenue décente ${ }^{21}$ dans laquelle ils nous posent ou qu'ils autorisent. Selon les heures du jour, les contextes, l'état d'esprit, nous nous asseyons diversement, observe Le Corbusier, poliment pour causer, actif pour pérorer, haut pour énoncer une thèse, béat pour ne rien faire. De la même façon que «le corset n'est plus», que le vêtement nouveau soutient la mobilité individuelle, l'homme moderne a désormais le droit de s'asseoir comme il le souhaite, comme il convient à « sa distinction».

Et cette aventure du mobilier se conclue par cet événement : sa disparition, au profit de l'idée d' "équipement domestique ». Parvenu ainsi au terme de la conférence, résumons : si formellement et constructivement, le vêtement moderne se trouve rapportée à une basique poche, il inspire par ailleurs tant pour sa matérialité, pour ses déclinaisons possibles, ses finitions, que pour le jeu d'accessoirisations et combinaisons, et encore pour la qualité d'élégance qui lui est incontestablement liée. Surtout, il est une référence de mobilité qui suggère à Le Corbusier une idéalité de maison vidée où «l'espace et la lumière abonde », ou l'on agit aisément et promptement. La liberté du corps permise par le nouveau vêtement emporte in fine vers une liberté d'esprit : «Voilà le fond du sac, penser à quelque chose ». Il se fait ainsi le modèle précurseur d'une libération de la vie «quand l'esprit de l'époque est encore recouvert de la défroque insupportable d'une époque mourante ${ }^{22}$.

\footnotetext{
${ }^{19}$ Les enjeux de la dispute sont plus complexes que cela comme Wigley l'explique. In White walls, designer dresses : the fashioning of modern architecture, 2nd ed. Cambridge (Massachusetts): MIT Press, 2001. pp. 147-149.

${ }^{20}$ Reich, Lilly : «Modefragen ». Die Form, ํ5, 1922. Cité et traduit in Stern, Radu : Against Fashion, Clothing as Art, 1850-1930, 2nd ed. Massachusetts : The MIT Press, 2004. p. 152.

${ }^{21}$ «L'aventure du mobilier ». Op.cit. p. 119.

${ }^{22}$ Le Corbusier : Vers une architecture, 1st ed Crès, 1923. Paris : Flammarion, 1995. p.II
} 


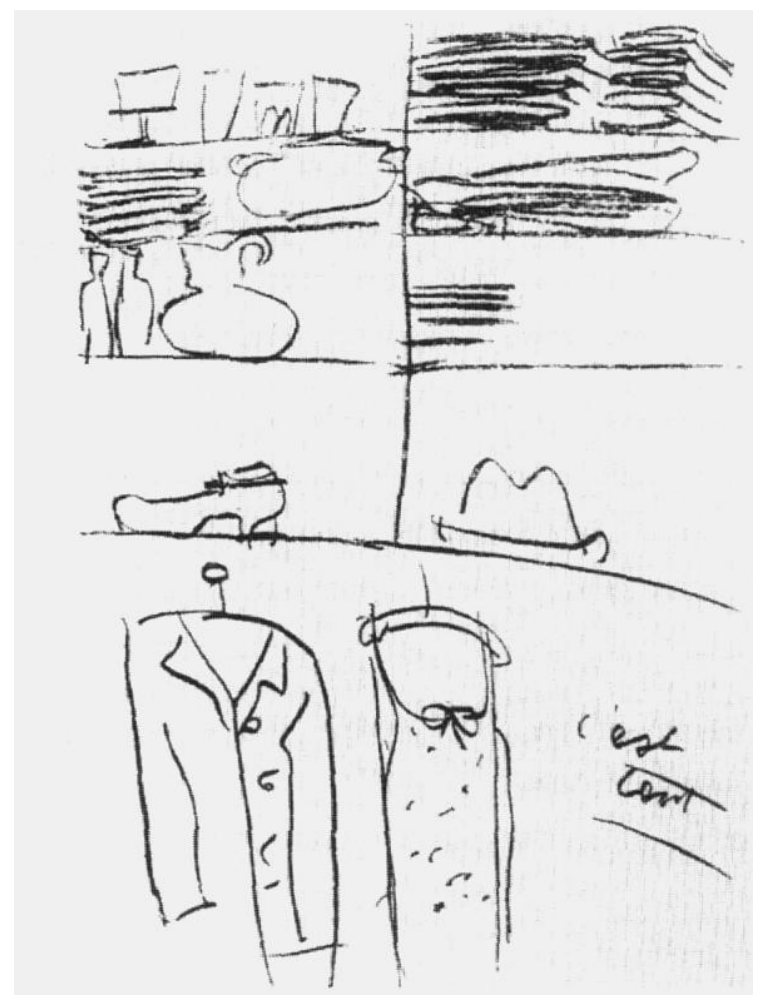

3. Croquis de Le Corbusier, 1929 CFLC/ADAGP

Les principes énoncés avaient en réalité déjà été expérimentés concrètement pour le Pavillon de l'Esprit Nouveau installé en 1925 lors de l'Exposition Internationale des Arts Décoratifs et Industriels Modernes à Paris. Exemple de «machine à habiter », il s'agissait de «montrer qu'un appartement peut être standardisé pour satisfaire aux besoins d'un homme de série » ${ }^{23}$, et «affirmer que l'architecture s'étend du moindre objet d'usage mobilier à la maison, à la ville et encore au-delà »; principe d'emboîtement, depuis le casier à la cellule d'habitation à son agrégat en colonie urbaine, auquel il convient d'insérer au préalable le vêtement.

\section{Sur le vêtement moderne : objet-membre ou enveloppe rythmique? Le Corbusier, designer de vêtement}

Cherchant dans des écrits préalables ce qui pourrait préciser cette mise en exergue du vêtement moderne, deux pistes retiennent l'attention.

En premier lieu, tel que présenté en 1923 dans Vers une architecture, le costume, ici masculin, apparait, en tête de liste, parmi les «objets-type » que l'époque a créés, formule qui devient en 1925 dans L'art décoratif d'aujourd'hui le leitmotiv de la révolution à mener contre l'expressivité artistique. Le vêtement y est introduit via le métier qui s'y rapporte, hors la mode et le stylisme : «chez un tailleur, un homme devant lui, et lui, un mètre à la main, prenant les mesures sur son homme. Nous revoici sur le plancher des vaches. Sérénité tonifiante des certitudes. $»^{24}$ Cette évocation permet d'insister sur l'échelle et la fonction humaines, garantes selon l'auteur d'une sauvegarde des besoins humains.

\footnotetext{
${ }^{23}$ Le Corbusier : «Pavillon de l'Esprit nouveau, Paris 1925 ». in Oeuvres complètes vol.1 : 1910-1929, Les éditions d'architecture (Artemis), Zurich, 1964, p. 98.

${ }^{24}$ Le Corbusier : L'art décoratif d'aujourd'hui. Paris : Flammarion, 1996. p. 70.
} 
L'argumentation s'apparente à celle de la conférence de 1929, mais le type tient la place du standard, et une raison évolutionniste se substitue à la raison industrielle qui emporte à énoncer le fameux concept d' «objetmembre artificiel $\gg$. La proposition étudiée ci-avant menant des vêtements, aux casiers, à la maison, se déploie ici dans une perspective de sélection organique dérivée de la théorie de Darwin. Dans un passage qui ferait référence à Samuel Butler, Le Corbusier explique comment les objets ne sont que de simples extensions des membres humains, poussées par l'évolution pour compléter les capacités naturelles et satisfaire de la sorte avec précision les besoins les plus objectifs. «Nous naissons nus et insuffisamment armés. Ainsi le creux de la main de Narcisse nous a conduit à inventer la bouteille ; le tonneau de Diogène qui constitue déjà une fameuse amélioration de nos organes de protection (notre peau, et notre cuir chevelu) nous donne la cellule primordiale de la maison ${ }^{25}$, et ainsi de suite, les livres prolongent notre mémoire, et les casiers dérivent d' «étuis », euxmêmes issus de la nécessité d'y ranger des «membres auxiliaires de garantie contre le froid ou le chaud»_sontce des vêtements? ? $^{26}$

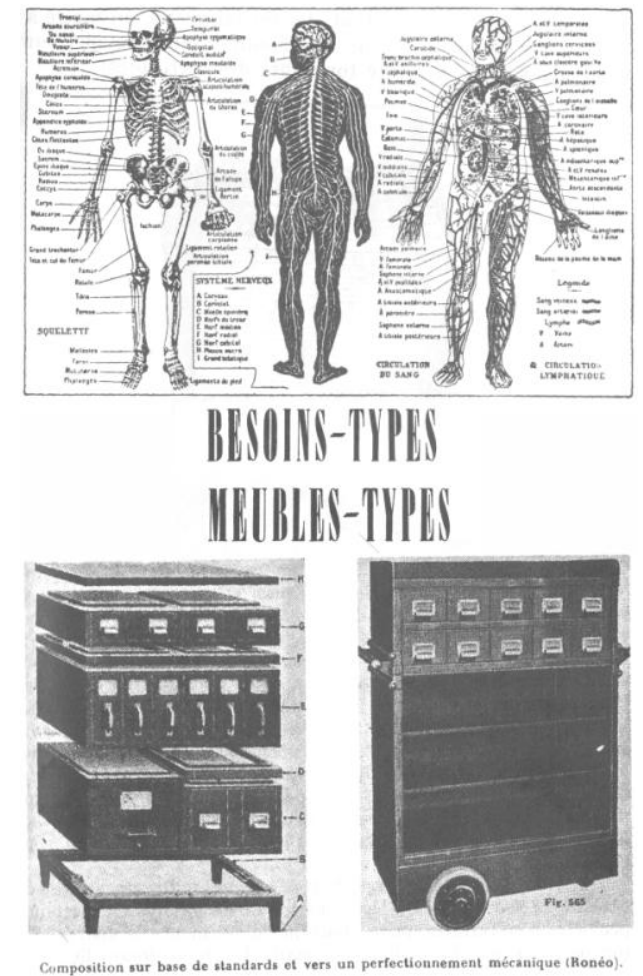

4. Illustrations extraites de L'art décoratif aujourd'hui, 1925 @FLC/ADAGP

Il est à noter que le rejet du décoratif ne signifie pas un rejet du vêtement. Si la toilette féminine est ici exposée comme suspecte pour son exubérance, et les modes comme douteuses, comme Wigler l'a analysé, le vêtementtype montre la voie à tous les objets utilitaires, à toutes les «machines » qu'appelle l'époque. Son origine est la peau, il est une peau supplémentaire ${ }^{27}$, et son horizon l'habitat minimum que le mythe de Diogène exemplarise. Débarrassé de son superflu, rendu objectif et docile, il est un habit qui permet de ne pas être habillé quand on ne peut demeurer nu dans le milieu «extra-humain », une sorte de vêtement de nudité pure qui confère au corps les

\footnotetext{
${ }^{25}$ Ibid. p. 72.

${ }^{26}$ A noter que l'artiste dada Raoul Hausmann, dans un article en 1924 pour le magazine Constructivist G, rapporta le vêtement à un «clothing object», le chapeau à «a head covering», et la mode à «the function of the body made visible». Cité par Radu Stern, in Against Fashion, Clothing as Art, 1850-1930. Op.cit. p.22.

${ }^{27}$ Métaphore faite par Le Corbusier dans un article pour une revue de psychologie en 1925. Cité par Wigley, op. cit. p.287
} 
prémices de la fonction domestique. Il doit en être ainsi de tout artefact : les artistes décoratifs sont assignés de cette mission orthopédique, activité finalement analogue à celle du tailleur, commente Le Corbusier, mais d'un tailleur moderne quand le client est pour lui un homme-type.

Cette théorie du vêtement-type comme premier objet-membre laisse toutefois dubitatif en regard de la multiplicité des qualités extrapolées du modèle vestimentaire moderne en 1929. Notamment, l'importance accordée au mouvement qu'il autorise est absente. Une autre source toutefois explicite cette préoccupation majeure, et constitue dans notre réflexion une seconde piste, même si le vêtement n'y apparait qu'indirectement.

En 1908, dans une lettre à sa mère, Charles-Edouard Jeanneret relate sa conception d'une sorte de multi-casier, «meuble fous-y-tout $»^{28}$, pour remédier à la situation ankylosante de sa «tôle d'étudiant » à Paris. Espace réduit de 3x4 mètres, il décrit comment il est obligé de glisser son corps, de sauter à saute-mouton à pieds joints, d'escalader, de rentrer son ventre, pour louvoyer entre la table, quelques chaises, un fauteuil, la malle qui sert de sofa, le lit, une baignoire-cuvette suspendue, et des habits accrochés à des clous. Las, il se fabriqua un mobilier combiné, pratique, «merveilleux» où tout trouve sa place, qui redonne au corps sa capacité à se mouvoir librement.

Le contexte semble annoncer le futur concept d'équipement domestique, et en effet, lors de la conférence, Le Corbusier rapportait que «l'anomalie du mobilier » l'obsède depuis une vingtaine d'années, qui justifie sa quête d'un casier «de mesure commune» pour contenir efficacement tous les objets, mais aussi «gagner une place considérable », et «circuler à l'aise ».

La relation exprimée ici entre l'aménagement mobilier et la "danse du corps dans l'espace intérieur ${ }^{29}$, peut être approchée et commentée grâce à la Rythmique développée par Emile Jaques-Dalcroze, à laquelle Le Corbusier fut initié par son frère Albert qui s'y exerçait. Anne Boissière relate comment cette théorie ancrée dans une pratique corporelle visant à réformer l'enseignement de la musique, trouve ses commencements expérimentaux au tournant du siècle dans la volonté de Dalcroze de contrer l' «arythmie $»^{30}$ de certains de ses élèves_l'anomalie mobilière citée par le Corbusier y fait-elle référence?_, soit le défaut d'équilibre dont un corps peut témoigner dans ses actions. S'il concerne ici l'apprentissage et l'exécution de la musique, ce problème toucha par ailleurs plus largement la problématique des modes de vie à l'ère industrielle accusés de mettre en défaut le rythme vivant par leurs cadences mécaniques. Symétriquement, Dalcroze s'appuie sur la capacité d'un organisme à s'informer de la musique et à la transformer en un rythme vécu qui se manifeste par un mouvement $v v^{3} \mathrm{t}^{31}$. La dimension synesthésique de la perception peut être exercée, afin d'atteindre une certaine dépersonnalisation subjective qui permet au corps de sentir profondément les combinaisons de rythme. Cet enjeu de la Rythmique la distingue d'autres tendances émancipatrices à cette époque cherchant l'expression passionnelle en phase avec les flux naturels.

Comme la notion de rythme, très débattue à l'époque, le terme mouvement n'est pas à comprendre au sens actuel. Mouvement recouvre une compréhension globale d'une énergie de vie, incluant autant les animations non visibles, comme la joie, changement intime du sentiment que l'on a de l'existence, que les motricités physiques

\footnotetext{
${ }^{28}$ Baudouï, Rémi ; Dercelles, Arnaud : Le Corbusier, Correspondances. Lettres à la famille, t.1 : 1900-1925. Infolio, 2011. Cité par Pitiot, Cloé : «Le Corbusier, Le mobilier corps et âme ». In Le Corbusier - Mesures de l'homme. Op. cit., p. 85.

${ }^{29}$ Ibid. p. 86

${ }^{30}$ Boissière, Anne : Musique Mouvement. Paris : Manucius, 2014. p. 77.

31 «Si la musique veut ordonner la mobilité du corps, elle doit s'informer, premièrement de ce que le corps attend d'elle. (...) Le corps abandonne donc sa vie propre à la musique pour la recevoir à nouveau de sa main, mais ordonnée et transfigurée ». Ibid. p.97
} 
comme les gestes ou déplacements. De même, la musique ne se résout pas en œuvres artistiques, elle est un phénomène qui fait partie intégrante de l'homme et s'active dans sa présence au monde, comme les travaux de psychologie phénoménologique de Erwin Straus le théorisaient.

Or, la Rythmique nous intéresse ici parce qu'elle se trouva des dispositifs idoines à son plein accomplissement. Les Espaces Rythmiques ${ }^{32}$ projetés par le scénographe Adolphe Appia en 1909-1910 témoignent d'une « complicité de l'espace » : l'existence d'un volume déterminé, de lumières et de conditions matérielles porteuses de «praticabilité ». Nous y reviendrons plus loin, notons pour le moment que l'aire de jeu en trois dimensions profite idéalement de lignes simples et de plots abstraits quoique aux mesures humaines qui sont « la paradoxale condition de la possibilité de la plasticité des corps mobiles et vivants $»^{33}$. Réciproquement, l'espace devient vivant pour celui qui le pratique. L'expérience de Le Corbusier dans sa chambre parisienne semble de cette nature.

En 1910, lors de son voyage en Orient, attestant de sa sensibilité pour de tels phénomènes, si ce n'est de son intérêt pour ces travaux, Charles-Edouard écrivait à ses parents au sujet du Parthénon : "Vous êtes pris, vous avez perdu le sens de l'échelle commune. Vous êtes assujetti à un rythme sensoriel (la lumière et le volume) et par des mesures habiles. ${ }^{34}$

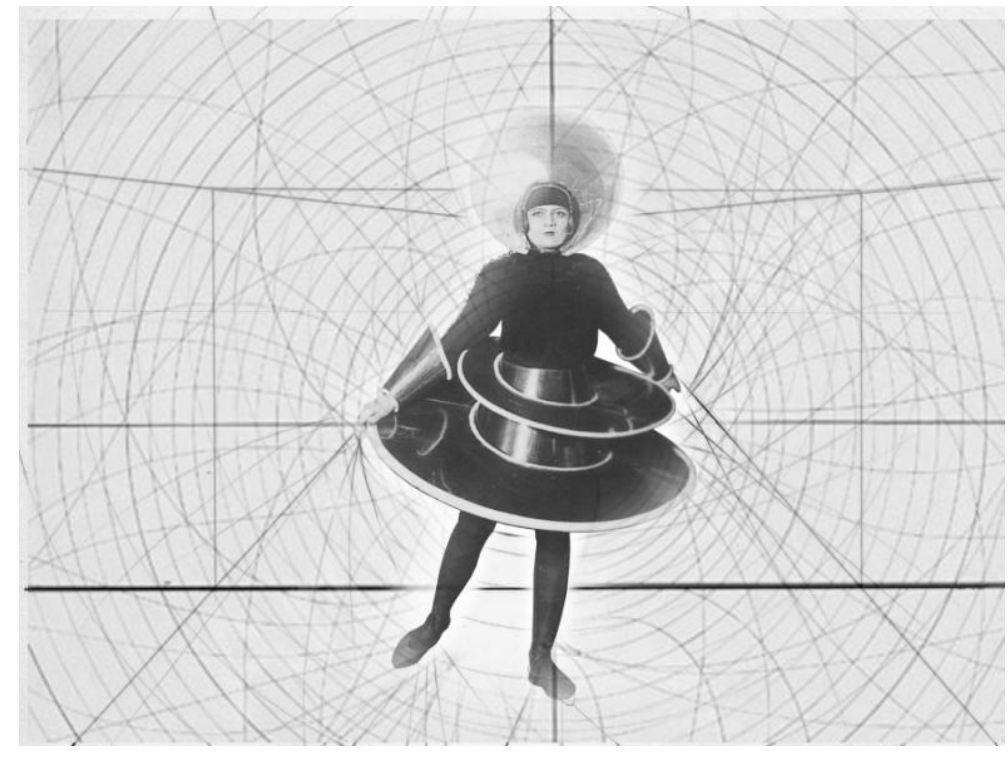

5. Danseuse du Ballet Triadique sur fonds de réseau spatial, d'après les dessins de Oskar Schlemmer

Dès lors, les vêtements modernes présentant ces qualités pourraient-ils recéler d'un potentiel d'enveloppes rythmiques? Cette question fut précisément travaillée au sein du Bauhaus, dans l'atelier de scène d'Oskar Schlemmer qui se fit le miroir des débats en vigueur. Le théâtre est pour lui un art de l'espace, soit «une structure de nombre et de mesures $»^{35}$ apte à simuler toute spatialité. Pour qu'il acquiert pleinement sa fonction, il faut lui conférer un rythme : «Le costume jouera ce rôle, à la fois comme support du mouvement, et pour luimême. L'acteur devient ainsi portion d'espace, portion d'architecture. »

\footnotetext{
${ }^{32}$ Certains dessins sont reproduits dans Appia, Adolphe : L'œuvre d'art vivant, Oeuvres complètes, t.3: 1906-1921. L'âge d'homme, Lausanne, 1988.

${ }^{33}$ Musique Mouvement. Op. cit. p. 93.

${ }^{34}$ Lettre à ses parents du 21 juin 1910, in BAUDOUÏ Rémi, DERCELLES Arnaud : Le Corbusier, Correspondances. Lettres à la famille, t.1 : 1900-1925. Op.cit. p. 311.

${ }^{35}$ Michaud, Eric : Théâtre au Bauhaus. Lausanne : L'âge d'homme-La cité, 1978. p. 128.
} 
Pour Le Ballet Triadique par exemple, il conçut des costumes aux volumétries géométrisées poussés à l'extrême d'une logique de vêtements-machines enveloppant et astreignant les corps organiques des danseurs, figurant de la sorte plastiquement la rencontre de l'homme naturel avec le monde industriel. Tel que Siegfried Giedion a commenté ce spectacle présenté en 1923 lors de la Semaine du Bauhaus _que visita Le Corbusier_, le costume rigide contraint en effet l'acteur à la discipline et aux gestes mécaniques, mais entraîne bientôt ses réactions, ses infléchissements, ses quêtes de nouveaux équilibres, et révèle bientôt une construction hybride spatio-temporelle inédite. Les mouvements s'aiguisent paradoxalement comme aucune machine ne le pourrait, constate l'historien. «De tous les domaines où s'exercent l'activité du Bauhaus, celui-là seul atteint à une parfaite liberté. » ${ }^{36} \mathrm{Ces}$ costumes, parfois agrémentés d'accessoires ou éléments scéniques furent pour Schlemmer des «praticables», autrement considérés comme « appareillage » du mouvement par Véronique Fabbri ${ }^{37}$.

Cet exemple atteste d'une certaine influence de dispositifs vestimentaires abstraitisés sur l'émergence d'un rythme ou mouvement vivant, qui va dans le sens de la possibilité de penser une transposition des dispositifs rythmiques de Appia au bénéfice du vêtement moderne. En retour, quand le vêtement moderne apparaît comme enveloppe rythmique, les casiers corbuséens se donnent à comprendre comme des «praticables » contribuant à offrir à l'espace domestique une certaine qualité rythmique.

Objet-membre ou enveloppe rythmique, les deux pistes ont en commun de loger la question du vêtement moderne au cœur des débats de l'époque sur la place de l'homme dans l'ère machiniste, et sur la question de la vie partagée entre approche scientifique et approche philosophique. Dans les années 1920, où la machine s'est immiscée dans toutes les dimensions du quotidien, le corps tend à être considéré comme un objet technique, la cinématographie permet d'en étudier les comportements, le taylorisme de les maitriser, la psychologie expérimentale d'en mesurer les seuils de perception.

Toutefois, ce qui distingue les deux points de vue se joue autour de la relation des vêtements (et derrière eux des artefacts) avec le corps, qui simule celle de la mécanique à l'organique. La relation est-elle de supplément, qui considère le corps comme incomplet, manquant de défense vis-à-vis des réalités du monde physique, et doit être protégé et complété? Tirant profit des connaissances biologiques, elle motive la maîtrise et la redéfinition de l'organique. Ou bien la relation est-elle d'appareillage, qui ménage un moyen intermédiaire de rencontre, confrontation, idéalement d'harmonie, entre le corps et la nature de plus en plus étrangère du contexte technique? Le vêtement comme enveloppe rythmique constitue « un appareillage extérieur, visible, qui ne fait que souligner l'appareillage ordinaire du corps, d'une manière discrète : ils ne se substituent pas au corps et aux gestes comme des prothèses ou des machines ${ }^{38}$ comme l'écrit Fabbri. Il ne fait qu'accompagner le corps organique dans son fonctionnement vivant.

Le vêtement moderne cristallise derechef une question idéologique majeure pour le design. En bref, doit-il porter? Ou être porté? Elargi du modèle à ses applications, la question devient : les artefacts modernes doivent-il engendrer un homme nouveau ou résulter de l'homme nouveau? Où le modèle du vêtement moderne emporte-t-il notre architecte?

\footnotetext{
${ }^{36}$ Ibid. p. 73.

${ }^{37}$ Fabbri, Véronique : "De la structure au rythme. L'appareillage des corps dans la danse". In Huyghe, Pierre-Damien (Dir.) : L'art au temps des appareils. Paris : L'Harmattan, 2005. pp. 93-121

${ }^{38}$ Ibid.p. 93
} 
Pour tenter de répondre, nous nous tournons vers Le Corbusier, designer de vêtement ${ }^{39}$. En 1952, pour la revue Formes et Vie, il présente un «costume pour la femme d'aujourd'hui », qu'il défend d'être « une création de haute-couture ${ }^{40}$. Il s'agit de vêtements «permanents », aux lignes simples (pièce d'étoffe carré sans coupe spéciale, robe d'une pièce juste plissée sous la taille ), de «dimensions proportionnées », pratique pour les activités de la vie quotidienne. De la sorte taillés pour une femme-type, ils satisfont à la définition d'un objettype par leur absence d'expression artistique. L'auteur précise même que ce costume est fait «pour un être vivant possédant une charpente osseuse, muscles et rondeurs utiles, chair et sveltesse » dont il a pensé, en architecte et peintre, le voilement ou dévoilement. Nous devons comprendre que le corps organique est bien à la base de la proposition_il faut un corps porteur_mais qu'elle n'entend pas pour autant offrir la condition de la nudité (le textile ne doit jamais serrer mais tomber bien, les jambes et bras nus visent la liberté de mouvement ) ni conférer l'apparence d'un autre corps, non plus suppléer à quelque fonction. Leur géométrie offre de laisser aller le corps ou de le soutenir, il incite une gestualité et permet in fine une attitude libre individuelle (qui peut aller jusque s'inventer de nouvelles manières de s'asseoir). La relation apparaît d'autant plus contrastée que ces vêtements sont de mesure vague par rapport aux contours corporels. A contrario, un vêtement prothèse comme une peau supplémentaire réduirait le jeu à néant.

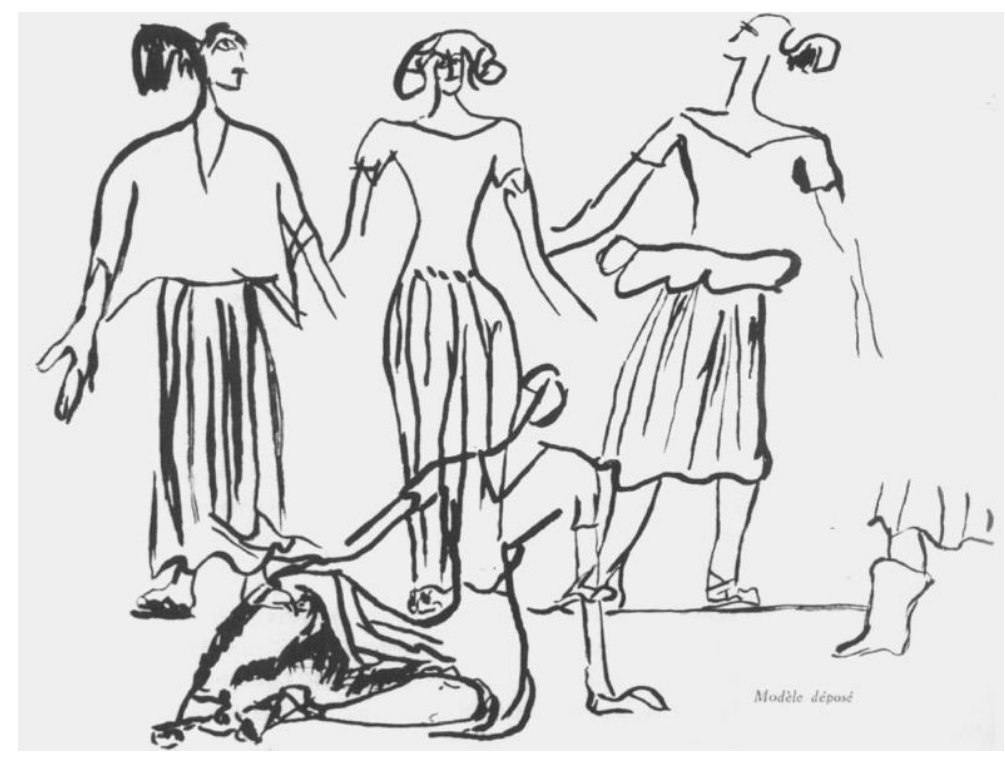

6. Dessin de Le Corbusier, Formes et Vie n², 1952 @FLC/ADAGDP

Le corps est couvert, disparaît majoritairement, mais se manifeste autrement au travers de cet objet, par son mouvement. La tenue se compose et se transforme par ailleurs au gré des envies «éphémères ». C'est dès lors une logique d'appareil qui se manifeste ici. Fabbri explique encore que «l'appareil ne forme pas, mais révèle l'être-forme du matériau (...) il n'a pas pour fonction de soumettre la matière à une forme, ni l'objet à un usage $»^{41}$. Ce costume ne conforme pas, ne porte pas, mais révèle l'être vivant derrière le corps organique.

La position de Le Corbusier n'est ainsi pas évidente, quand ce projet de vêtement témoigne du potentiel du vêtement type à se faire enveloppe rythmique.

\footnotetext{
${ }^{39}$ Le projet s'en tient en l'occurrence à des dessins et commentaires, une indication « Modèle déposé » ne renvoie à aucune information à l'INPI.

${ }^{40}$ Le Corbusier : "Note". Formes et Vie, N², 1952. Paris : Éditions Falaize. p.11.

41 "De la structure au rythme. L'appareillage des corps dans la danse". Op.cit. p. 96.
} 


\section{Sur la conception prothétique corbuséenne du design . Le tailleur-designer}

Selon Forty, quand il analyse les rouages historiques du développement du design industriel, nous avons affaire, avec la théorie des objets-membres, à une conception prothétique du design ${ }^{42}$ que Le Corbusier aurait majoritairement contribué à diffuser.

L'idée d'un parallèle, littéral ou métaphorique, entre le processus d'évolution des objets et celui du monde naturel se développe au XIXème siècle dans le contexte des sciences biologiques naissantes; et profite alors dialectiquement autant à préciser la compréhension du vivant qu'à orienter le déploiement économique du monde artificiel. Une idée de l'organique infiltre à la fin du siècle les discours sur l'art ou l'architecture. Par-delà l'acception théorique que Sullivan ou Wright lui donneront, Muthesius y puisera même en 1902 pour expliquer les variations de la mode ${ }^{43}$. Malgré la diversité et l'imprécision scientifique des extrapolations, malgré le démenti apporté par la génétique quant à la réalité et la pertinence d'un tel schème, la théorie prothétique aura, dans ses diverses versions, une fortune remarquable au XXème siècle et encore actuellement.

Réagissant à la version promue par Le Corbusier, qui laisse imaginer que la conception des objets se situe en dehors des questions poétiques et esthétiques, repliée sur une venue naturelle au monde, Forty remarque que cette thèse ne vaut rien puisqu'elle situe l'acte de design en dehors de la conscience humaine, laissant planer le plus grand mystère. Malgré tout, l'historien pointe que cette idée va faire son chemin dans la discipline et donner prise à un fonctionnalisme radical. Elle invite en effet à considérer le processus créatif d'objet comme la seule traduction de la mécanique corporelle en un produit mécanique artificiel, adossée sur l'étude la plus rigoureuse et exhaustive des dimensionnements et logiques mécaniques de l'être humain. C'est en effet une perspective prise par le design à partir des années 1950 en s'appuyant sur l'ergonomie, comme en témoignent Joe et Josephine, les deux américains modèles que Henry Dreyfuss a conçu comme charte de design ${ }^{44}$. Plus récemment, les modélisations algorythmiques des phénomènes naturels, les biotechniques et biomimétiques, les processus évolutionnaires, etc. actualisent cette conception à l'ère numérique ${ }^{45}$.

Quoique le discours approche $« d u$ moins superficiellement ${ }^{46}$ un tel point de vue, Forty défend Le Corbusier de telles ambitions. Aux côtés des «objets-sentiments» des Beaux-Arts, des «objets à réaction poétique » de la nature, les «objets-membres » illustrent métaphoriquement la quête d'objets-type parfaits fondée davantage sur des questions de pureté esthétique de leur forme, telle que les lois de la nature en génèrent pour les coquillages ou les fleurs, que sur une gestion industrielle de la standardisation des formes des produits en relation aux corps. Par ailleurs, les illustrations de L'art décoratif d'aujourd'hui révèlent assez qu'il n'existe aucune référence formelle, loin s'en faut, au biomorphisme.

Puis l'auteur explique ce qu'il estime des applications prometteuses du concept prothétique. Elles sont de deux natures : d'abord sociale, liée à la nature symbolique de tout objet prothèse du fait de sa présence autour du corps et son influence sur l'habitus ; ensuite sensorielle ou auto-érotique, liée aux contacts corporels et à la

\footnotetext{
${ }^{42}$ Forty, Adrian : «Industrial design and prosthesis ». Ottagono, N ${ }^{\circ} 16$, Septembre 1990. Milano : CO.P.IN.A. pp. 114-129

${ }^{43}$ «On peut sans doute expliquer les raisons psychologiques de ce changement dans le fait que certains organes activés en admirant telle forme deviennent las avec le temps et retrouve une activité en s'orientant vers d'autres formes». MUTHESIUS, Hermann : "Die Moderne Umbildung Unserer Äesthetischen Anschauungen (1902). In Kultur und Kunst, Jena : Berlegt Bei Eugen Diederichs, 1909. p.35-75, citation p. 39. Traduction personnelle.

${ }^{44}$ Dreyfuss, Henry : Designing for people (1955), 4th ed. New York : Alworth Press, 2012.

${ }^{45}$ Steadman, Philip : The evolutions of design : biological analogy in architecture and the applied arts, 2nd ed. Abingdon (UK) - New York (USA) : Routledge, 2008.

46 «Industrial design and prosthesis ». Op.cit. . Traduction in Midal, Alexandra : Design, l'anthologie. Saint-Etienne : Cité du design - Genève : HEAD, 2013. p. 398.
} 
participation gestuelle. De tels objets convoquent des qualités esthétiques étendues, capables de suppléer d'une autre façon à des manques du corps que la psychanalyse corrobore. Il faut y voir un nouvel idéal de luxe, suggère l'historien, non plus défini, comme c'est le cas habituellement par des valeurs propres au travail artisanal ou artistique, mais «c'est à cela, à la qualité de l'expérience que l'objet procure aux sens, que la vision prothétique $d u$ design attache le plus de valeur ${ }^{47}$. Notons que l'ergonomie tient compte de tels critères esthétiques et psychologiques dans ses «facteurs humains » pourvu qu'on puisse les rapporter à des données (Joe et Joséphine ont des «inhibitions et obsessions $»^{48}$, sont sensibles à un toucher inconfortable, à une couleur agressive ou à une odeur désagréable, etc.).

Bien que Forty ait recours abondamment à des vêtements et accessoires pour étayer sa démonstration, dont un modeste chapeau qui nous rappelle la panoplie-type de notre architecte, Forty ne l'inclue pas dans ces ouvertures du prothétique. Telle que nous l'avons approchée, la position de Le Corbusier ne se retrouve pas en effet dans cette vision du design comme prothèse, quoiqu'elle se définisse bien par un rapport privilégié entre corps et objet. Je voudrais montrer qu'elle ne se résout pas en un formalisme adossé sur des «mesures de l'homme » prises avec le mètre du tailleur, et soutenir a contrario que l'inscription fondatrice du vêtement moderne, (et il faut rappeler qu'il s'agit du vêtement moderne féminin), c'est-à-dire le fait que le vêtement moderne soit la base idéologique de tout objet-membre et maison-cellule, permet d'entrevoir et d'esquisser un point de vue prothétique alternatif aux versions fonctionnaliste, ergonomique et même néo-luxe, riche d'autres mesures et sensations proprement humaines. Cette proposition n'est pas sans conséquence quand on se souvient que le Modulor fut commenté et critiqué comme anthropo-métrique (stricto sensu la caractérisation des «mesures de l'homme ») déshumanisante ${ }^{49}$.

Pour amener cela, nous nous intéressons aux propos que Le Corbusier tient sur la conception à l'âge de la machine lorsqu'il voyage aux USA en 1935, et y découvre une situation avancée sur le plan industriel qui le laisse dubitatif. L'ouvrage qui le relate, Quand les cathédrales étaient blanches, débute ainsi : «Je vais montrer par l'USA, pris comme exemple, que les temps sont neufs mais que la maison est inhabitable $»^{50}$. On y trouve un appel réitéré à la réforme du costume masculin, le vêtement moderne acquérant même un statut paradigmatique dans un passage explicite.

«Curieuse fin de civilisation : l'homme qui porta des plumes d'autruche sur la tête, roses, blanches et bleu-roi, une vêture de brocarts ou de soie rutilante, ne sait plus que fourrer ses deux mains dans les poches d'un pantalon noir. (...) Il demeure que la question est à reprendre et que la transformation du costume masculin est nécessaire. ${ }^{51}$ C'est pour lui une question éthique et institutionnelle. Comme Wigley le révèle, face à l'influence du vêtement et derrière lui au spectre inquiétant de la mode, le mouvement moderne développa un système de défense et traça sa voie sous la surveillance de «Watchdogs » ${ }^{52}$ qui s'assurèrent, à tout le moins, de garder le vêtement féminin à distance. Seul le vêtement standard masculin eut droit de considération officiellement (et les murs blancs comme surfaces-type prothétiques en sont l'écho). Le vestiaire féminin, moderne y compris,

\footnotetext{
${ }^{47}$ Ibid. p.400.

${ }^{48}$ Ibid. p. 194.

49 «Mégalomanie (...) perte de réalité humaine (...) isolement de la nature (...) Dans ce milieu extrêmement conformé, l'homme ne peut être admis qu'anthropologiquement : le Modulor est la fiche anthropométrique. ». Commentaires de Hervé Baley et Dominique Zimbacca parus dans le numéro spécial de Aujourd'hui : art et architecture en 1965 dédié à l'architecte juste décédé.

${ }^{50}$ Le Corbusier : Quand les cathédrales étaient blanches. Paris : Plon, 1937. p.7.

${ }^{51}$ Ibid. p. 124.

${ }^{52}$ White walls. Un chapitre est explicitement dédié à la « Fashion Policy». Op.cit. pp. 35-57.
} 
cristallisant les forces transitoires, changeantes, incontrôlables du monde dont on cherchait à se prémunir ; quand c'est justement ce qui offre à Le Corbusier d'y percevoir « l'esprit de l'âge machiniste », un certain ordre vivant. Il répète d'ailleurs dans son texte l'épanouissement des femmes, comme il le fera de nouveau en 1942 dans son Entretien aux étudiants en architecture, où le vêtement féminin moderne est chargé de ses arguments éthiques sur la modernité.

«Le costume est l'expression d'une civilisation. (...) Le costume décèle les sentiments les plus fondamentaux ; par lui, nous manifestons notre dignité, notre distinction, notre frivolité ou nos ambitions profondes. Bien que standard, le costume masculin ne se dérobe pas à l'intervention individuelle. Mais il ne convient plus. De ce qu'il subsiste, nous avons la preuve que la révolution machiniste n'es pas à maturité. » ${ }^{53}$

Plus loin, dans ce même ouvrage, un passage sur le jazz retient notre attention en ce que Le Corbusier semble y remarquer cette même capacité que le vêtement à traduire le renouveau et à porter la réforme. Le jazz, commente-t-il, est un événement (comme il avait commenté la disparition du mobilier en 1929). Il ne résulte pas d'une conception délibérée. Il représente «les forces présentes » qui se manifestent via les corps accompagnés, « appareillés », de leurs instruments. Nous nous trouvons dans la même situation que le corps appareillé par le vêtement, mais ici la mécanique de l'orchestre (qu'il apparente à une turbine) ancre plus directement le dispositif dans l'ère industrielle. Or, comme l'auteur en témoigne personnellement, il autorise une expérience sensuelle et thymique. «Musique d'époque de construction : novatrice. Elle inonde le corps et le coeur. (...) Elle est si puissante, si irrésistible psycho-physiologiquement, qu'elle nous a arrachsé à la passivité de l'audition, et nous fait danser ou gesticuler, participer. " ${ }^{54}$ Et encore "La musique entre dans la poitrine des hommes et des femmes, s'y rive, y emporte le flux du sang, y met en dynamisme le corps entier, tandis que la pensée s'élève sur l'aile de la mélodie. » ${ }^{55}$

Ce passage illustre le potentiel de la machine, capable de mobiliser profondément les individus, en libérant des énergies originaires. En 1942, il vantera de nouveau les femmes aux vêtements libérés (dont il souligne les couleurs comme signe vital) pour leur démarche vive et saine, leur audace et leur optimisme. Ce sont précisément de tels mouvements (spontanés, animés ou dansés, ou le fait qu'une attitude exprime de la timidité ou de la confiance, de l'insécurité ou, de la stabilité) qui motivèrent les travaux de Straus, en ce qu'ils échappaient (et échappent toujours, signale Boissière) à l'explication scientifique, à la causalité déterministe et aux modélisations objectives. Quand la locomotion et la motricité étaient sujets de tous les intérêts, de tels élans demeurent la seule affaire de celui qui les vit. On peut certes les mesurer, les décomposer et les mimer, (même dans leurs aspects sensoriels), mais on ne sait pas les susciter, on ne parvient pas en reproduire artificiellement la sensation ou «sentiment vivant». Ils se replient en effet sur la catégorie philosophique du «sentir». Et la Rythmique constitue en quelque sorte une méthode pour sentir, (re)trouver et exercer ce qui rejoint le «rythme vécu ».

Or, quelque-chose dans le témoignage de Le Corbusier nous y renvoie. Il attire en effet l'attention sur la discordance entre les mouvements libérés, gesticulatoires, et «la mathématique » de la musique jazz. Derrière les corps emportés, sang et âme, il relève avec insistance «l'exactitude implacable », l'orchestre «impeccable, sans trou, régulier, en ascension rythmique $»^{56}$. Un ordre se loge paradoxalement derrière l'expérience de

\footnotetext{
${ }^{53}$ Quand les cathédrales étaient blanches. Op.cit. p. 124-125

${ }^{54}$ Ibid. p. 180

${ }^{55}$ Ibid. p. 181

${ }^{56}$ Ibid. p. 183
} 
saisissement. De même, comme nous l'avions évoqué rapidement, une géométrie épurée est la paradoxale condition de l'activation plastique des corps. Les Espaces Rythmiques sont, comme le commente Boissière, « d'une étonnante immobilité et apparente vacuité » 57.

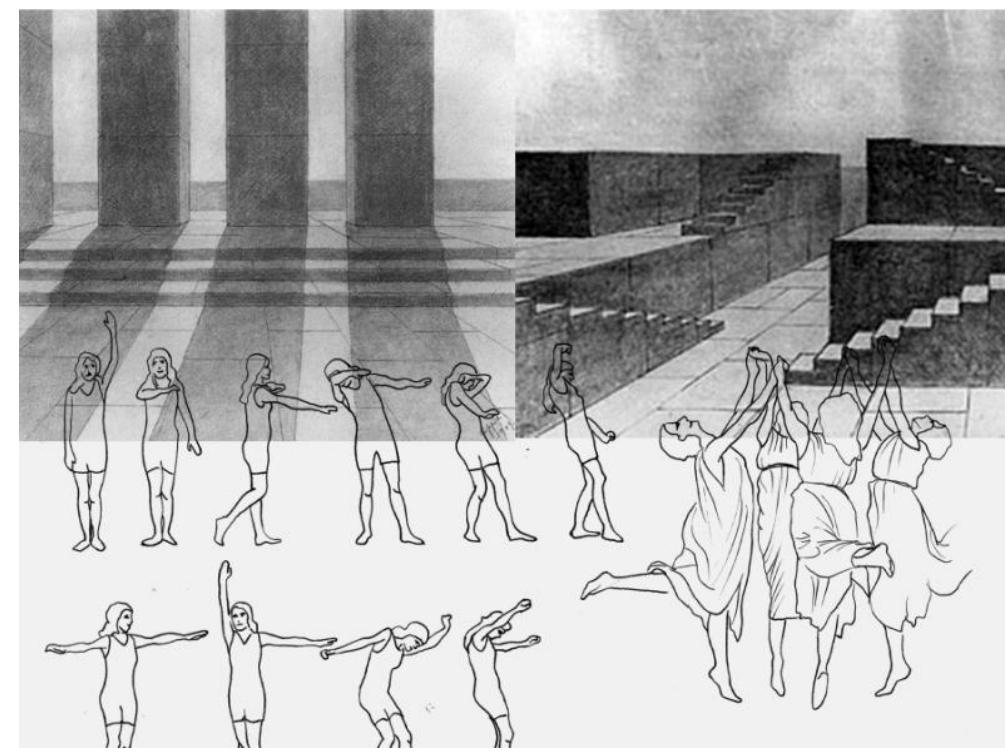

7. Exercices de Rythmique, d'après la Méthode Jacques-Dalcroze,

sur fonds des dessins Espaces rythmiques de Adolphe Appia, 1909-1910

Ils ne sont pas eux-mêmes animés dans leurs dessins. On n'y trouve aucune évocation organique telle que ondulation, serpentine ou entrelac. A contrario, leur composition est régulière, à partir de lignes essentiellement horizontales et verticales, qui se concentrent localement en des escaliers créant une logique ascendante. Elle n'a pas pour fonction de guider le mouvement, mais de fournir tout appui utile pour qu'il s'élance lui-même (comme on s'appuie sur un pilier pour prendre une impulsion). Il n'est pas donné par le dispositif, le corps n'est pas porté. Et ce contexte autorise par contraste l'émergence d'un mouvement ou rythme propre. Le sentiment vivant résulte de cette mobilisation profonde du corps appareillé par un ordre étranger à lui-même.

Un tel dispositif évoque une qualité prothétique dont la condition est la paradoxale dissimilitude avec le corps organique (il n'est pas besoin, au contraire, qu'il soit ergonomique), et dont l'expérience spécifique se situe au niveau de ces sensations d'être vivant. Le vêtement moderne, le costume féminin designed by Le Corbusier, le jazz, et après eux, les objets-type corbuséens, illustrent cette prothétique paradoxale.

De là, comme Straus l'a théorisé, naît une sensation d'espace qui outrepasse l'espace physique, un espace présentiel (ou «espace acoustique »). Celui-ci se définit par une notion subtile de mesure, qui n'a à voir ni avec le mètre mécanique, ni avec le tempo musical, mais qui peut être comprise comme cadre ou envergure au sein duquel on ressent l'espace vivant, et que l'on peut seulement rapporter à des «proportions » ${ }^{58}$. C'est bien le corps qui porte, en lui, autour de lui, cette sorte d'espace. Comme Boissière l'analyse, elle peut être comprise dans le cadre de la philosophie de Heidegger, comme un saut qualitatif, de l'ordre du poétique, qui est la condition de l'habiter.

\footnotetext{
${ }^{57}$ Musique Mouvement. Op.cit. p.76

58 «La mesure serait-elle en nous-même? Serions-nous le créateur de l'espace? Pour qui? Nous sommes seuls. Ce sera donc pour nous seul que nous créerons l'espace, c'est-à-dire des proportions que notre corps pourra mesurer dans l'espace sans limite qui lui échappe. » Ibid. p.115
} 
La prothétique corbuséenne nous apparaît dès lors comme un travail de tailleur-designer à qui revient la tâche de concevoir des artefacts comme des enveloppes, de «mesure moyenne » (à l'instar des casiers standards) ou «de dimensions proportionnées » (à l'instar de son projet vestimentaire), fonctionnels au sens de praticables, avec le moyen desquelles le corps pourra transformer les phénomènes complexes ambiants de l'époque (sa «musique ») en un mouvement vivant, et développer son habitation. Comme il en existe des versions extensive ou supplétive, c'est là une prothétique poétique.

\section{Conclusion}

«Pour parler de Le Corbusier, il faut remonter aux années 20. Là, il traça tout son chemin. Quarante ans après, il restait dans ses sillons » ${ }^{59}$, commentait Iannis Xenakis en 1965.

Notre rapide parcours autour de la considération de Le Corbusier pour le vêtement moderne ne le dément pas, quand il révèle comment celui-ci se mua à ce moment-là en un modèle fondateur. La conférence de 1929 tient à ce titre un rôle important quand elle synthétise ce qui l'occupait diffusément sur le sujet depuis de nombreuses années, et parvient à en extraire la substance active, la mobilité, au-delà des raisons formelles et fonctionnelles.

Surtout, attestant d'une position hardie au sein du mouvement moderne et du territoire du design en émergence, elle ose focaliser l'attention sur le nouveau vestiaire féminin, ce qui représente une précision par rapport aux écrits théoriques précédents qui avaient posé le costume standard masculin à l'origine d'une idéologie prothétique, nonobstant le scientisme ou l'imprécision qui cerne cette conception. Le féminin se comprend ici, non pas au sens de la féminité et de la mode, mais comme «une forme de l'esthétique qui convient » à «l'esprit de l'âge machiniste », l'idéalité d'une résolution entre ordonnancement et plasticité (comme il le relèvera en 1935 pour le jazz), permanence et éphémère (comme il ne notera en 1952 au sujet de la «femme d'aujourd'hui »), qui précise l'importance accordée au mouvement harmonieux ou gracieux. Il est la musique ou le rythme des temps modernes, au sens que l'on a dit. Tout le propos de la conférence en est insidieusement affecté, et les «objetstype » ou «machines» y sont vantés nouvellement pour l'espace de jeu qu'ils autorisent, soit comme des praticables ou « appareils».

Toutefois, la richesse du discours tenu ce jour-là et l'inscription de la problématique au cœur des débats ne s'éclairent qu'avec le recours de techniques et théories propres à l'époque, telle que la Rythmique et la phénoménologie du mouvement, les notions d'œuvre vivante, de sensation et de mesure. Il atteste de la sorte de son ancrage vif et pertinent dans une actualité _ il n'est pas banal, il n'est pas universel_ et subit consécutivement le risque d'être amoindri le jour suivant. Et en effet, la notion d'équipement domestique à laquelle il aboutit représente aujourd'hui un pont entre objets-membres et maison-cellule, omettant la référence initiale. Sa prothétique par ailleurs se retrouve évaluée en regard d'une technoscientifisation croissante du vivant.

Mais cette conférence constitue également un appel au design à repenser le costume masculin, c'est-à-dire à généraliser la réforme moderne initiée. Et Le Corbusier réitérera cette injonction en 1937 de retour des USA, en 1942 s'adressant aux étudiants ... Appel sans réponse, quoique force est de constater qu'il participe ici-même au réveil d'une pensée du vêtement en design.

\footnotetext{
${ }^{59}$ Aujourd'hui : art et architecture, $\mathrm{N}^{\circ} 51$ spécial, Novembre 1965. Op.cit. p.94.
} 


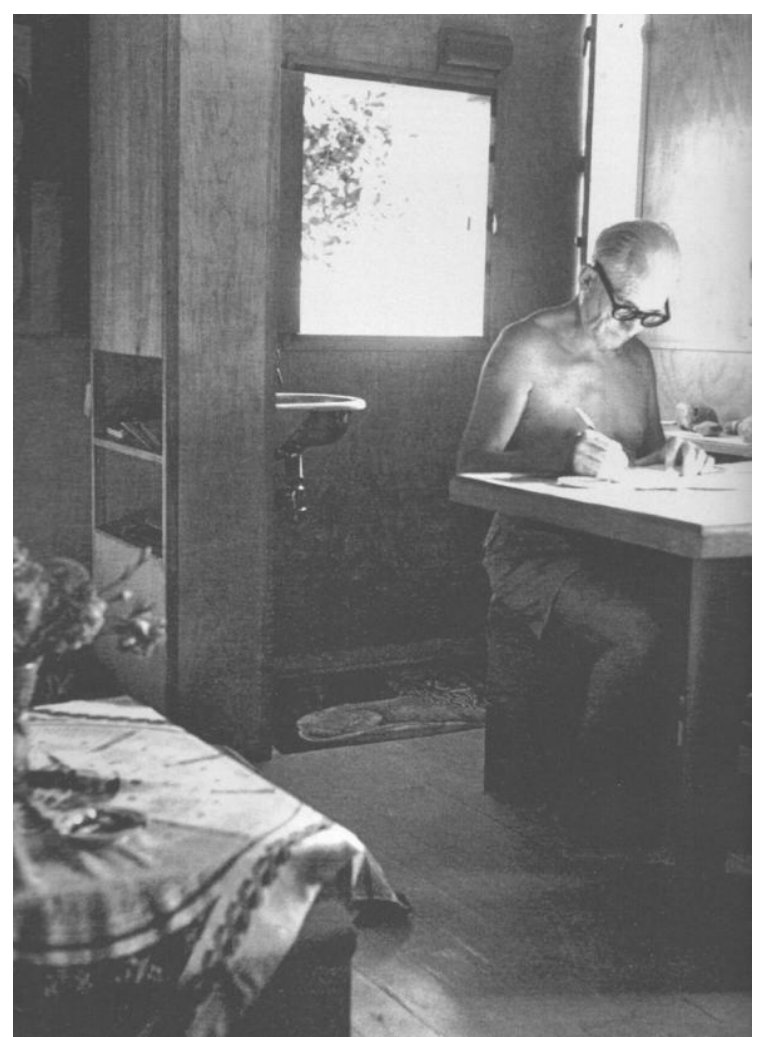

8. Le Corbusier dans le Cabanon, photo Lucien Hervé 1952 @ FLC/ADAGDP

En 1952, Le Corbusier installe son cabanon à Roquebrune-Cap-Martin, conçue selon l'outil de mesure que représente le Modulor. De dimensions très proches de sa chambre d'étudiant de 1908 (3,66m x3,66m x2,66m ), les casiers et le mobilier y sont optimisés, favorisant l'usage et la pratique d'un espace rigoureux, essentiellement vide, animé simplement de quelques lignes et de la lumière naturelle. «Application révélatrice. (...) La mise en service de cette construction a dépassé tous les espoirs. ${ }^{60}$ commente-t-il sommairement. Il y vivra quasiment $\mathrm{nu}$, se plaît-on à répéter. Notre hypothèse est que cette maison, plus petite qu'une cellule d'habitation, plus vague qu'un vêtement-type, constitue pour lui un pyjama architectural $^{61}$, soit, à l'instar du vêtement moderne, une enveloppe rythmique qu'il put exercer corporellement et ressentir comme une architecture vivante.

\section{Références bibliographiques}

Banham, Peter Reyner : Théorie et design à l'ère industrielle. Orléans : édition HYX, 2009.

Boissière, Anne : Musique Mouvement, Paris : Manucius, 2014.

Cinqualbre, Olivier; Migayrou, Fréderic (Dir.) : Le Corbusier - Mesures de l'homme, Paris : Editions du Centre Pompidou, 2015. Catalogue de l'exposition tenue du 29 avril au 3 août 2015 au Centre Pompidou à Paris.

Fabbri, Véronique : "De la structure au rythme. L'appareillage des corps dans la danse". In Huyghe, PierreDamien (Dir.) : L'art au temps des appareils. Paris : L'Harmattan, 2005. pp. 93-121

\footnotetext{
${ }^{60}$ Le Corbusier : Oeuvres complètes, vol.5 : 1946-1952. Zurich : Les Editions d'architecture - Editions Gisberger, 1953. p. $62-63$

${ }^{61}$ Référence à la formule choisie par Gottfried Semper lors de sa dernière conférence tenue à Zurich en 1869 lorsqu'il encourage la jeune génération d'architectes à concevoir enfin le nouvel « habit architectural » digne de prolonger la nouvelle idée de l'histoire telle qu'il leur transmet. in Semper Gottfried : Du style et de l'architecture. Marseille : Parenthèses, Marseille, 2007. p.360
} 
Forty, Adrian : «Industrial design and prosthesis ». Ottagono, $\mathrm{N}^{\circ} 16$, septembre 1990. Milano : CO.P.IN.A. pp. 114-129

Guillaume, Valérie (Dir) : Europe 1910-1933, Quand l'art habillait le vêtement. Paris : Edition Paris Musées, 1997.

Jeanneret, Charles-Edouard; OZENFANT, Amédée : Après le cubisme. Paris : Éditions des Commentaires, 1918.

Le Corbusier : "Note”. Formes et Vie, N², 1952. Paris : Éditions Falaize. p.11.

Le Corbusier : «Entretien avec les étudiants des écoles d'architecture » (1942). in La Charte d'Athènes. Paris : Éditions du Seuil, 1971.

Le Corbusier : L'Art décoratif d'aujourd'hui, $1^{\text {st }}$ ed Crès, 1925. Paris : Flammarion, 1996.

Le Corbusier : Précisions sur un état présent de l'architecture et de l'urbanisme. Paris : Edition Altamira, 1994. Fac-similé de l'éd. de Paris : Crès, 1930.

Le Corbusier : Quand les cathédrales étaient blanches. Paris : Plon, 1937.

Le Corbusier : Vers une architecture, 1st ed Crès, 1923. Paris : Flammarion, 1995,

Loos, Adolf : Paroles dans le vide - Malgré tout. Paris : Editions Ivrea, 1994.

Michaud, Eric : Théâtre au Bauhaus. Lausanne : L'âge d'homme-La cité, 1978.

Midal, Alexandra : Design, l'anthologie. Saint-Etienne : Cité du design - Genève : HEAD, 2013.

Monneyron, Frédéric (Dir.) : Vêtement et littérature. Perpignan : Presses Universitaires de Perpignan, 2001.

Petit, Jean : Le Corbusier parle. Paris : Editions Forces Vives, 1967.

Semper Gottfried : Du style et de l'architecture. Marseille : Parenthèses, Marseille, 2007. Traduction et préface de Jacques Soulillou.

Steadman, Philip : The evolutions of design : biological analogy in architecture and the applied arts, 2nd ed. Abingdon (UK) - New York (USA) : Routledge, 2008.

Stern, Radu : Against Fashion, Clothing as Art, 1850-1930, 2nd ed. Massachusetts : The MIT Press, 2004.

Valéry, Paul : Oeuvres I. Paris : La Pléiade, 1957.

Wigley, Mark : White walls, designer dresses : the fashioning of modern architecture, 2nd ed. Cambridge (Massachusetts) - London (England) : MIT Press, 2001.

Xenakis, Iannis : w/o title. Aujourd'hui : art et architecture, $\mathrm{N}^{\circ} 51$ spécial, Novembre 1965 . Boulogne-sur-seine : w/o pub. p.94. 